\title{
Continuous dependence on data for a solution of the quasilinear parabolic equation with a periodic boundary condition
}

\author{
Fatma Kanca ${ }^{1 *}$ and Irem Sakinc Baglan ${ }^{2}$
}

*Correspondence:

fatma.kanca@khas.edu.tr

'Department of Information

Technologies, Kadir Has University, Istanbul, 34083, Turkey

Full list of author information is

available at the end of the article

\section{Abstract}

In this paper we consider a parabolic equation with a periodic boundary condition and we prove the stability of a solution on the data. We give a numerical example for the stability of the solution on the data.

\section{Introduction}

Consider the following mixed problem:

$$
\begin{aligned}
& \frac{\partial u}{\partial t}-\frac{\partial^{2} u}{\partial x^{2}}=f(x, t, u), \quad(x, t) \in D:=\{0<t<T, 0<x<\pi\} \\
& u(0, t)=u(\pi, t), \quad t \in[0, T], \\
& u_{x}(0, t)=u_{x}(\pi, t), \quad t \in[0, T], \\
& u(x, 0)=\varphi(x), \quad x \in[0, \pi]
\end{aligned}
$$

for a quasilinear parabolic equation with the nonlinear source term $f=f(x, t, u)$.

The functions $\varphi(x)$ and $f(x, t, u)$ are given functions on $[0, \pi]$ and $\bar{D} \times(-\infty, \infty)$ respectively. Denote the solution of problem (1)-(4) by $u=u(x, t)$. The existence, uniqueness and convergence of the weak generalized solution of problem (1)-(4) are considered in [1]. The numerical solution of problem (1)-(4) is considered [2].

In this study we prove the continuous dependence of the solution $u=u(x, t)$ upon the data $\varphi(x)$ and $f(x, t, u)$. In [3], a similar iteration method is used with this kind of a local boundary condition for a nonlinear inverse coefficient problem for a parabolic equation. Then we give a numerical example for the stability.

\section{Continuous dependence upon the data}

In this section, we will prove the continuous dependence of the solution $u=u(x, t)$ using an iteration method. The continuous dependence upon the data for linear problems by different methods is shown in $[4,5]$.

Theorem 1 Under the following assumptions, the solution $u=u(x, t)$ depends continuously upon the data.

(0) 2013 Kanca and Baglan; licensee Springer. This is an Open Access article distributed under the terms of the Creative Commons Attribution License (http://creativecommons.org/licenses/by/2.0), which permits unrestricted use, distribution, and reproduction in any medium, provided the original work is properly cited. 
$\left(\mathrm{A}_{1}\right)$ Let the function $f(x, t, u)$ be continuous with respect to all arguments in $\bar{D} \times(-\infty, \infty)$ and satisfy the following condition:

$$
|f(t, x, u)-f(t, x, \tilde{u})| \leq b(x, t)|u-\tilde{u}|,
$$

where $b(x, t) \in L_{2}(D), b(x, t) \geq 0$,

$\left(\mathrm{A}_{2}\right) f(x, t, 0) \in C^{2}[0, \pi], t \in[0, \pi]$,

$\left(\mathrm{A}_{3}\right) \varphi(x) \in C^{2}[0, \pi]$.

Proof Let $\phi=\{\varphi, f\}$ and $\bar{\phi}=\{\bar{\varphi}, \bar{f}\}$ be two sets of data which satisfy the conditions $\left(\mathrm{A}_{1}\right)$ $\left(\mathrm{A}_{3}\right)$.

Let $u=u(x, t)$ and $v=v(x, t)$ be the solutions of problem (1)-(4) corresponding to the data $\phi$ and $\bar{\phi}$ respectively, and

$$
|f(t, x, 0)-\bar{f}(t, x, 0)| \leq \varepsilon \quad \text { for } \varepsilon \geq 0 .
$$

The solutions of (1)-(4), $u=u(x, t)$ and $v=v(x, t)$, are presented in the following form, respectively:

$$
\begin{aligned}
& u_{0}(t)=\varphi_{0}+\frac{2}{\pi} \int_{0}^{t} \int_{0}^{\pi} f(\xi, \tau, A u(\xi, \tau)) d \xi d \tau \\
& u_{c k}(t)=\varphi_{c k} e^{-(2 k)^{2} t}+\frac{2}{\pi} \int_{0}^{t} \int_{0}^{\pi} f(\xi, \tau, A u(\xi, \tau)) e^{-(2 \pi k)^{2}(t-\tau)} \cos 2 k \xi d \tau, \\
& u_{s k}(t)=\varphi_{s k} e^{-(2 k)^{2} t}+\frac{2}{\pi} \int_{0}^{t} \int_{0}^{\pi} f(\xi, \tau, A u(\xi, \tau)) e^{-(2 \pi k)^{2}(t-\tau)} \sin 2 k \xi d \tau .
\end{aligned}
$$

Let $A u(\xi, \tau)=\frac{u_{0(\tau)}}{2}+\sum_{k=1}^{\infty}\left[u_{c k}(\tau) \cos 2 k \xi+u_{s k}(\tau) \sin 2 k \xi\right]$.

$$
\begin{aligned}
& v_{0}(t)=\bar{\varphi}_{0}+\frac{2}{\pi} \int_{0}^{t} \int_{0}^{\pi} \bar{f}(\xi, \tau, A v(\xi, \tau)) d \xi d \tau \\
& v_{c k}(t)=\bar{\varphi}_{c k} e^{-(2 k)^{2} t}+\frac{2}{\pi} \int_{0}^{t} \int_{0}^{\pi} \bar{f}(\xi, \tau, A v(\xi, \tau)) e^{-(2 \pi k)^{2}(t-\tau)} \cos 2 k \xi d \tau \\
& v_{s k}(t)=\bar{\varphi}_{s k} e^{-(2 k)^{2} t}+\frac{2}{\pi} \int_{0}^{t} \int_{0}^{\pi} \bar{f}(\xi, \tau, A v(\xi, \tau)) e^{-(2 \pi k)^{2}(t-\tau)} \sin 2 k \xi d \tau .
\end{aligned}
$$

Let $A v(\xi, \tau)=\frac{v_{0}(\tau)}{2}+\sum_{k=1}^{\infty}\left[v_{c k}(\tau) \cos 2 k \xi+v_{s k}(\tau) \sin 2 k \xi\right]$.

From the condition of the theorem, we have $u^{(0)}(t)$ and $v^{(0)}(t) \in B$. We will prove that the other sequential approximations satisfy this condition.

$$
\begin{aligned}
& u_{0}^{(N+1)}(t)=u_{0}^{(0)}(t)+\frac{2}{\pi} \int_{0}^{t} \int_{0}^{\pi} f\left(\xi, \tau, A u^{(N)}(\xi, \tau)\right) d \xi d \tau, \\
& u_{c k}^{(N+1)}(t)=u_{c k}^{(0)}(t)+\frac{2}{\pi} \int_{0}^{t} \int_{0}^{\pi} f\left(\xi, \tau, A u^{(N)}(\xi, \tau)\right) e^{-(2 k)^{2}(t-\tau)} \cos 2 k \xi d \tau, \\
& u_{s k}^{(N+1)}(t)=u_{s k}^{(0)}(t)+\frac{2}{\pi} \int_{0}^{t} \int_{0}^{\pi} f\left(\xi, \tau, A u^{(N)}(\xi, \tau)\right) e^{-(2 k)^{2}(t-\tau)} \sin 2 k \xi d \tau, \\
& v_{0}^{(N+1)}(t)=v_{0}^{(0)}(t)+\frac{2}{\pi} \int_{0}^{t} \int_{0}^{\pi} \bar{f}\left(\xi, \tau, A v^{(N)}(\xi, \tau)\right) d \xi d \tau,
\end{aligned}
$$




$$
\begin{aligned}
& v_{c k}^{(N+1)}(t)=v_{c k}^{(0)}(t)+\frac{2}{\pi} \int_{0}^{t} \int_{0}^{\pi} \bar{f}\left(\xi, \tau, A v^{(N)}(\xi, \tau)\right) e^{-(2 k)^{2}(t-\tau)} \cos 2 k \xi d \tau, \\
& v_{s k}^{(N+1)}(t)=v_{s k}^{(0)}(t)+\frac{2}{\pi} \int_{0}^{t} \int_{0}^{\pi} \bar{f}\left(\xi, \tau, A v^{(N)}(\xi, \tau)\right) e^{-(2 k)^{2}(t-\tau)} \sin 2 k \xi d \tau,
\end{aligned}
$$

where $u_{0}^{(0)}(t)=\varphi_{0}, u_{c k}^{(0)}(t)=\varphi_{c k} e^{-(2 k)^{2} t}, u_{s k}^{(0)}(t)=\varphi_{s k} e^{-(2 k)^{2} t}$ and $v_{0}^{(0)}(t)=\bar{\varphi}_{0}, v_{c k}^{(0)}(t)=$ $\bar{\varphi}_{c k} e^{-(2 k)^{2} t}, v_{s k}^{(0)}(t)=\bar{\varphi}_{s k} e^{-(2 k)^{2} t}$.

First of all, we write $N=0$ in (6)-(7). We consider $u^{(1)}(t)-v^{(1)}(t)$

$$
\begin{aligned}
u^{(1)}(t)-v^{(1)}(t)= & \frac{u_{0}^{(1)}(t)-v_{0}^{(1)}(t)}{2} \\
& +\sum_{k=1}^{\infty}\left[\left(u_{c k}^{(1)}(t)-v_{c k}^{(1)}(t)\right)+\left(u_{s k}^{(1)}(t)-v_{s k}^{(1)}(t)\right)\right] \\
= & \left.\varphi_{0}-\overline{\varphi_{0}}\right) \\
& +\frac{2}{\pi} \int_{0}^{t} \int_{0}^{\pi}\left[f\left(\xi, \tau, A u^{(0)}(\xi, \tau)\right)-\bar{f}\left(\xi, \tau, A v^{(0)}(\xi, \tau)\right)\right] d \xi d \tau \\
& +\left(\varphi_{c k}-\overline{\varphi_{c k}}\right) e^{-(2 k)^{2} t} \\
& +\frac{2}{\pi} \int_{0}^{t} \int_{0}^{\pi}\left[f\left(\xi, \tau, A u^{(0)}(\xi, \tau)\right)-\bar{f}\left(\xi, \tau, A v^{(0)}(\xi, \tau)\right)\right] \\
& \times e^{-(2 \pi k)^{2}(t-\tau)} \cos 2 \pi k \xi d \xi d \tau+\left(\varphi_{s k}-\overline{\varphi_{s k}}\right) e^{-(2 k)^{2} t} \\
& +\frac{2}{\pi} \int_{0}^{t} \int_{0}^{\pi}\left[f\left(\xi, \tau, A u^{(0)}(\xi, \tau)\right)-\bar{f}\left(\xi, \tau, A v^{(0)}(\xi, \tau)\right)\right] \\
& \times e^{-(2 \pi k)^{2}(t-\tau)} \sin 2 \pi k \xi d \xi d \tau .
\end{aligned}
$$

Adding and subtracting

$$
\begin{aligned}
& \int_{0}^{t} \int_{0}^{\pi} f(\xi, \tau, 0) d \xi d \tau, \quad \int_{0}^{t} \int_{0}^{\pi} e^{-(2 k)^{2}(t-\tau)} f(\xi, \tau, 0) \cos 2 \pi k \xi d \xi d \tau \\
& \int_{0}^{t} \int_{0}^{\pi} e^{-(2 k)^{2}(t-\tau)} f(\xi, \tau, 0) \sin 2 \pi k \xi d \xi d \tau
\end{aligned}
$$

to both sides and applying the Cauchy inequality, Hölder inequality, Lipschitz condition and Bessel inequality to the right-hand side of (8) respectively, we obtain

$$
\begin{aligned}
\left|u^{(1)}(t)-v^{(1)}(t)\right| \leq & 2\left|u_{0}^{(1)}(t)-v_{0}^{(1)}(t)\right|+4 \sum_{k=1}^{\infty}\left(\left|u_{c k}^{(1)}(t)-v_{c k}^{(1)}(t)\right|+\left|u_{s k}^{(1)}(t)-v_{s k}^{(1)}(t)\right|\right) \\
\leq & \|\varphi-\bar{\varphi}\| \\
& +\left(\frac{\sqrt{3 T}+\pi}{\sqrt{6} \pi}\right)\left(\int_{0}^{t} \int_{0}^{\pi} b^{2}(\xi, \tau) d \xi d \tau\right)^{\frac{1}{2}}\left|\bar{u}^{(0)}(t)\right| \\
& +\left(\frac{\sqrt{3 T}+\pi}{\sqrt{6} \pi}\right)\left(\int_{0}^{t} \int_{0}^{\pi} \bar{b}^{2}(\xi, \tau) d \xi d \tau\right)^{\frac{1}{2}}\left|\bar{v}^{(0)}(t)\right| \\
& +\left(\frac{\sqrt{3 T}+\pi}{\sqrt{6} \pi}\right)\left(\int_{0}^{t} \int_{0}^{\pi} f^{2}(\xi, \tau, 0)-\bar{f}^{2}(\xi, \tau, 0) d \xi d \tau\right)^{\frac{1}{2}},
\end{aligned}
$$




$$
\begin{aligned}
A_{T}= & \|\varphi-\bar{\varphi}\|+\left[\left(\frac{\sqrt{3 T}+\pi}{\sqrt{6} \pi}\right)\|b(x, t)\|\left|\bar{u}^{(0)}(t)\right|+\left(\frac{\sqrt{3 T}+\pi}{\sqrt{6} \pi}\right)\|b(x, t)\|\left|\bar{v}^{(0)}(t)\right|\right] \\
& +\left(\frac{\sqrt{3 T}+\pi}{\sqrt{6} \pi}\right)\|f-\bar{f}\|, \\
\|\varphi-\bar{\varphi}\| & =\max \frac{\left|\varphi_{0}-\overline{\varphi_{0}}\right|}{2}+\sum_{k=1}^{\infty} \max \left|\varphi_{c k}-\overline{\varphi_{c k}}\right|+\max \left|\varphi_{s k}-\overline{\varphi_{s k}}\right| .
\end{aligned}
$$

For $N=1$,

$$
\begin{aligned}
\left|u^{(2)}(t)-v^{(2)}(t)\right| \leq & \frac{\left|u_{0}^{(2)}(t)-v_{0}^{(2)}(t)\right|}{2}+\sum_{k=1}^{\infty}\left(\left|u_{c k}^{(2)}(t)-v_{c k}^{(2)}\right|+\left|u_{s k}^{(2)}(t)-v_{s k}^{(2)}(t)\right|\right) \\
\leq & \left(\frac{\sqrt{3 T}+\pi}{\sqrt{6} \pi}\right)\left(\int_{0}^{t} \int_{0}^{\pi} b^{2}(\xi, \tau) d \xi d \tau\right)^{\frac{1}{2}} A_{T} \\
& +\left(\frac{\sqrt{3 T}+\pi}{\sqrt{6} \pi}\right)\left(\int_{0}^{t} \int_{0}^{\pi} \bar{b}^{2}(\xi, \tau) d \xi d \tau\right)^{\frac{1}{2}} A_{T} .
\end{aligned}
$$

For $N=2$,

$$
\begin{aligned}
\left|u^{(3)}(t)-v^{(3)}(t)\right| & \frac{\left|u_{0}^{(3)}(t)-v_{0}^{(3)}(t)\right|}{2}+\sum_{k=1}^{\infty}\left(\left|u_{c k}^{(3)}(t)-v_{c k}^{(3)}(t)\right|+\left|u_{s k}^{(3)}(t)-v_{s k}^{(3)}(t)\right|\right) \\
\leq & \left(\frac{\sqrt{3 T}+\pi}{\sqrt{6} \pi}\right)\left(\int_{0}^{t} \int_{0}^{\pi} b^{2}(\xi, \tau)\left|\bar{u}^{(2)}(t)-\bar{v}^{(2)}(t)\right|^{2} d \xi d \tau\right)^{\frac{1}{2}} \\
& +\left(\frac{\sqrt{3 T}+\pi}{\sqrt{6} \pi}\right)\left(\int_{0}^{t} \int_{0}^{\pi} \bar{b}^{2}(\xi, \tau)\left|\bar{u}^{(2)}(t)-\bar{v}^{(2)}(t)\right|^{2} d \xi d \tau\right)^{\frac{1}{2}} \\
\leq & \left(\frac{\sqrt{3 T}+\pi}{\sqrt{6} \pi}\right)^{2} A_{T}\left[\int_{0}^{t} \int_{0}^{1} b^{2}(\xi, \tau)\left(\int_{0}^{\tau} \int_{0}^{\pi} b^{2}\left(\xi_{1}, \tau_{1}\right) d \xi_{1} d \tau_{1}\right) d \xi d \tau\right]^{\frac{1}{2}} \\
& +\left(\frac{\sqrt{3 T}+\pi}{\sqrt{6} \pi}\right)^{2} A_{T}\left[\int_{0}^{t} \int_{0}^{1} b^{2}(\xi, \tau)\left(\int_{0}^{\tau} \int_{0}^{1} \bar{b}^{2}\left(\xi_{1}, \tau_{1}\right) d \xi_{1} d \tau_{1}\right) d \xi d \tau\right]^{\frac{1}{2}} \\
\leq & \left(\frac{\sqrt{3 T}+\pi}{\sqrt{6} \pi}\right)^{2} A_{T} \frac{1}{\sqrt{2}}\left[\left(\int_{0}^{t} \int_{0}^{1} b^{2}(\xi, \tau) d \xi d \tau\right)^{2}\right]^{\frac{1}{2}} \\
& +\left(\frac{\sqrt{3 T}+\pi}{\sqrt{6} \pi}\right)^{2} A_{T} \frac{1}{\sqrt{2}}\left[\left(\int_{0}^{t} \int_{0}^{1} \bar{b}^{2}(\xi, \tau) d \xi d \tau\right)^{2}\right]^{\frac{1}{2}} .
\end{aligned}
$$

In the same way, for a general value of $N$, we have

$$
\begin{aligned}
\left|u^{(N+1)}(t)-v^{(N+1)}(t)\right| \leq & \frac{\left|u_{0}^{(N+1)}(t)-v_{0}^{(N+1)}(t)\right|}{2} \\
& +\sum_{k=1}^{\infty}\left(\left|u_{c k}^{(N+1)}(t)-v_{c k}^{(N+1)}(t)\right|+\left|u_{s k}^{(N+1)}(t)-v_{s k}^{(N+1)}(t)\right|\right) \\
\leq & A_{T} \cdot a_{N}=a_{N}\left(\|\varphi-\bar{\varphi}\|+C(t)+M_{1}\|f-\bar{f}\|\right),
\end{aligned}
$$


where

$$
\begin{aligned}
a_{N}= & \left(\frac{\sqrt{3 T}+\pi}{\sqrt{6} \pi}\right)^{N} \frac{A_{T}}{\sqrt{N !}}\left[\left(\int_{0}^{t} \int_{0}^{\pi} b^{2}(\xi, \tau) d \xi d \tau\right)^{2}\right]^{\frac{N}{2}} \\
& +\left(\frac{\sqrt{3 T}+\pi}{\sqrt{6} \pi}\right)^{N} \frac{A_{T}}{\sqrt{N !}}\left[\left(\int_{0}^{t} \int_{0}^{\pi} \bar{b}^{2}(\xi, \tau) d \xi d \tau\right)^{2}\right]^{\frac{N}{2}}
\end{aligned}
$$

and

$$
M_{1}=\left(\frac{\sqrt{3 T}+\pi}{\sqrt{6} \pi}\right)^{N} .
$$

(The sequence $a_{N}$ is convergent, then we can write $a_{N} \leq M, \forall N$.)

It follows from the estimation ([1, pp.76-77]) that $\lim _{N \rightarrow \infty} u^{(N+1)}(t)=u(t)$.

Then let $N \rightarrow \infty$ for the last equation

$$
|u(t)-v(t)| \leq M\|\varphi-\bar{\varphi}\|+M_{2}\|f-\bar{f}\|
$$

where $M_{2}=M \cdot M_{1}$.

If $\|f-\bar{f}\| \leq \varepsilon$ and $\|\varphi-\bar{\varphi}\| \leq \varepsilon$, then $|u(t)-v(t)| \leq \varepsilon$.

\section{Numerical example}

In this section we consider an example of numerical solution of (1)-(4) to test the stability of this problem. The numerical procedure of (1)-(4) is considered in [2].

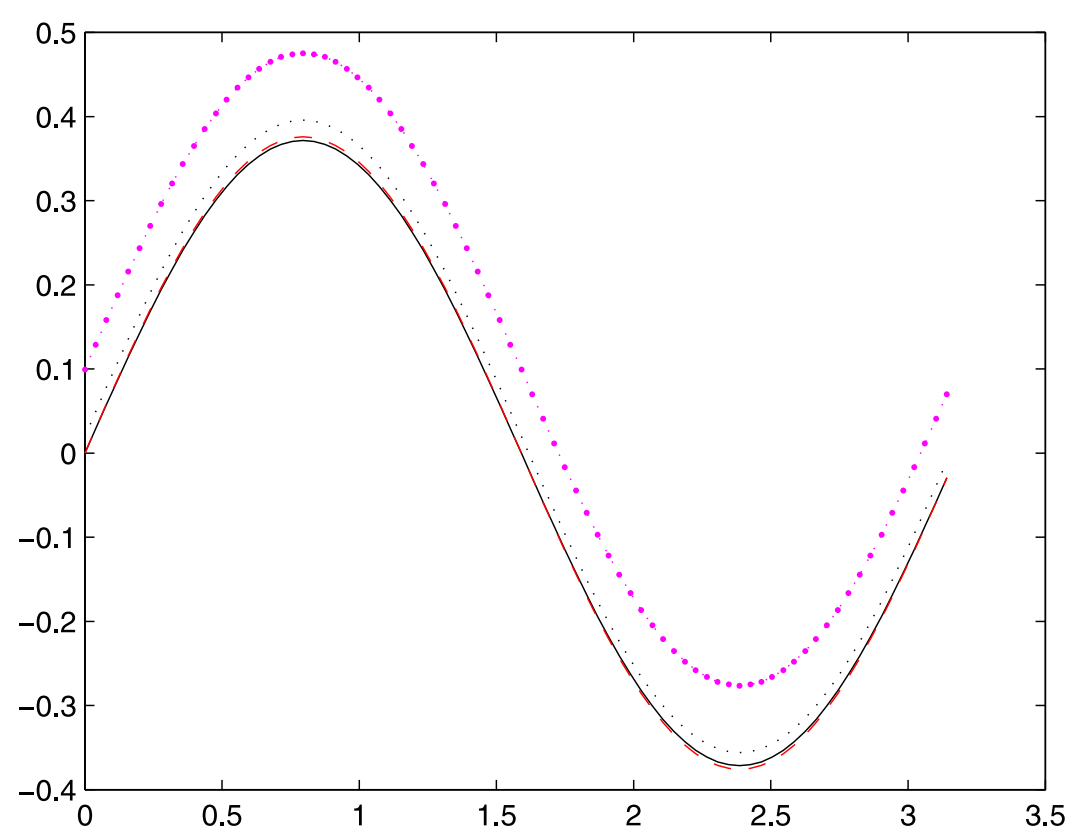

Figure 1 The exact and numerical solutions of $u(x, 1)$. The exact and numerical solutions of $u(x, 1),(-)$ for $\varepsilon=0$, (-.) for $\varepsilon=0.05$, (..) for $\varepsilon=0.01$, the exact solution is shown with a dashed line. 
Example 1 Consider the problem

$$
\begin{aligned}
& \frac{\partial u}{\partial t}-\frac{\partial^{2} u}{\partial x^{2}}=3 u, \\
& u(x, 0)=\sin 2 x, \quad x \in[0, \pi], \\
& u(0, t)=u(\pi, t), \quad t \in[0, T], \quad u_{x}(0, t)=u_{x}(\pi, t), \quad t \in[0, T] .
\end{aligned}
$$

It is easy to see that the analytical solution of this problem is

$$
u(x, t)=\sin 2 x \exp (-t)
$$

In this example, we take $f(x, t, u)=f(x, t, u)+\varepsilon$ and $\varphi(x)=\varphi(x)+\varepsilon$ for different $\varepsilon$ values. The comparisons between the analytical solution and the numerical finite difference solution for $\varepsilon=0,01, \varepsilon=0,05$ values when $T=1$ are shown in Figure 1 .

The computational results presented are consistent with the theoretical results.

Competing interests

The authors declare that they have no competing interests.

\section{Authors' contributions}

FK conceived the study, participated in its design and coordination and prepared computing section. ISB participated in the sequence alignment and achieved the estimation.

\section{Author details}

'Department of Information Technologies, Kadir Has University, Istanbul, 34083, Turkey. ${ }^{2}$ Department of Mathematics, Kocaeli University, Kocaeli, 41380, Turkey.

\section{Acknowledgements}

Dedicated to Professor Hari M Srivastava.

Received: 7 January 2013 Accepted: 29 January 2013 Published: 14 February 2013

\section{References}

1. Ciftci, I, Halilov, H: Fourier method for a quasilinear parabolic equation with periodic boundary condition. Hacet. J. Math. Stat. 37, 69-79 (2008)

2. Sakinc, I: Numerical solution of the quasilinear parabolic problem with periodic boundary condition. Hacet. J. Math. Stat. 39, 183-189 (2010)

3. Cannon, J, Lin, Y: Determination of a parameter $p(t)$ in holder classes for some semilinear parabolic equations. Inverse Probl. 4, 595-606 (1988)

4. Cannon, J, Lin, Y, Wang, S: Determination of a control parameter in a parabolic partial differential equation. J. Aust. Math. Soc. Ser. B, Appl. Math 33, 149-163 (1991)

5. Kanca, F, Ismailov, M: Inverse problem of finding the time-dependent coefficient of heat equation from integral overdetermination condition data. Inverse Probl. Sci. Eng. 20, $463-476$ (2012) 